\title{
MELANOSIS OF THE IRIS AND NEW FORMATION OF A HYALINE MEMBRANE ON ITS SURFACE
}

\author{
BY \\ D. J. WOOD \\ CAPE TOWN
}

IN the end of July, 1925, I was consulted by a lady, Mrs. H. H., with a view to getting new glasses. She was sixty-three, had a little astigmatism, and with correction saw $6 / 6$ with her right eye and $6 / 5$ with the left.

The left eye, however, showed several peculiarities. The pupil was oval and eccentric upwards; the iris was in contact with the lens on the inner side only, and there was a large pigmented plaque on the iris below. The iris was dark greyish blue in colour.

Examination with the corneal microscope showed other abnormalities. On the outer side the iris was raised up in a ridge which apparently reached the cornea, there were a few dark specks on the cornea below, not like precipitates; there were some remains of pigment on the lens suggestive of persistent pupillary membrane, and there was coronary cataract. The most curious finding, however, was that all over the outer half of the iris, from its root to the edge of the pupil, there were large numbers of rounded eminences, brown in colour, and many of them raised sufficiently to cast definite shadows. The upper margin of the pupil showed a large uveal eversion, and even on this there were several nodules. There were none visible, however, on the pigmented plaque below. The endothelium, examined under a high power, showed no changes and the aqueous was absolutely clear. Nothing in the way of a growth could be seen on the outer side between the iris and the lens, though one could see as far as to the ciliary processes.

I spent some time in making a picture of the condition, though the patient was easily tired, and not enthusiastic. It illustrates fairly well the appearance when I saw her first. She disappeared until April, 1926, and returned then because of visual failure in the left eye. The cornea had become somewhat steamy, tension was higher than in the right eye, and fine details of the iris were obscured. Vision was only 6/9. One could see that there was no cupping of the disc, and the field of vision was full, but there was no doubt of the presence of glaucoma. There was also no doubt that the prominences on the iris had advanced further round, both above and below.

Eserine brought down the tension, and the cornea cleared, but the patient again ceased attendance, perhaps because I had begun to think ill of the eye and told her so. In August she returned 


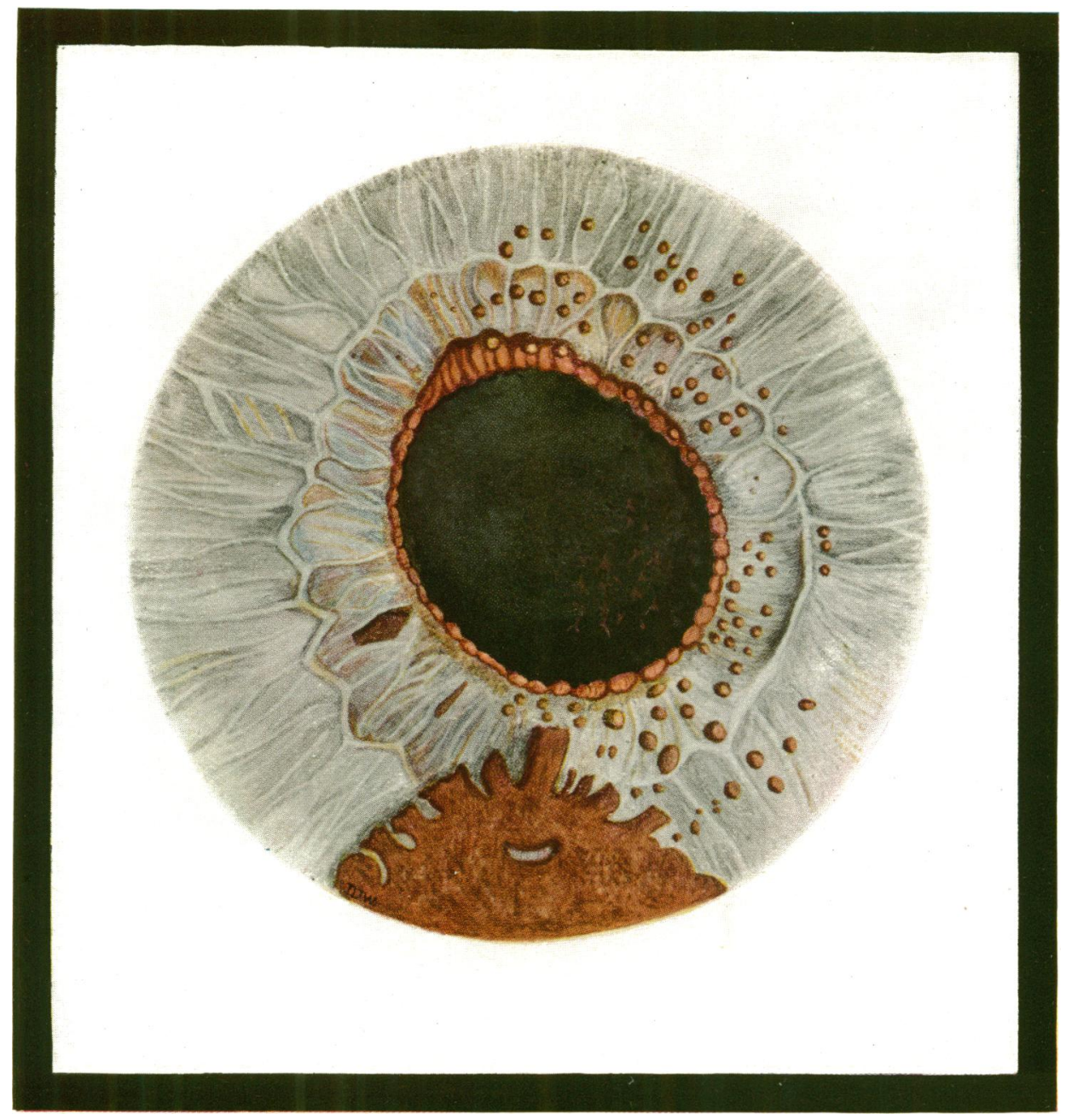

W

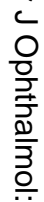

竞

뭉 
with not merely increased tension but also some pain. Vision had gone down to $6 / 12$. The nodules had spread all round the iris, and there was a bulge forwards in it above, similar to that on the outer side. It seemed difficult not to believe that one was dealing with a malignant condition, and one which did not justify any glaucoma operation. After much delay and consultations with all my colleagues and her friends she agreed to part with the eye and it was removed in November, sixteen months after her first visit. Unfortunately the eyeball was placed for several hours in an empty bottle, instead of the one containing Bouin's solution, so that it became somewhat collapsed. No tumour was found in any part of the eye and the posterior half was normal. Sections were cut in celloidin, some were bleached, and most were stained with haematoxylin and eosin.

Many abnormalities were found. The iris was overlaid with a hyaline membrane and endothelium continuous with Descemet's membrane, and extending over the edge of the pupil, being very visible where it covered the ectropion. At the site where the iris was adherent to the cornea the hyaline was thickened (Fig. 8), and not only did it pass over the pupillary edge, but it extended as far back as the position of the adhesion, viz., nearly half-way across the uveal surface.

The angle of the anterior chamber was closed by the fusion of the two membranes in many sections, and in most Schlemm's canal was well behind the apparent angle of the anterior chamber. In the latter there were frequently seen clusters of hexagonal endothelial cells, and balls of pigment which when bleached showed sometimes a nucleus, but sometimes became mere ghosts of cells (Figs. 6 and 7 ).

The prominences on the iris were much more numerous than the intra vitam appearances suggested. Some sections contained only two or three, but upon others one could see up to a dozen between the iris root and pupillary edge. Some were composed of only a few cells, but most were larger and some were as high as half the thickness of the iris. Some lay flat on the surface, but most were raised up like golf balls on a tee (Figs. 1 and 2), and in a few one could see that the cluster of cells came through a distinct stoma in the hyaline membrane.

The outer cells of the nodules were rounded, with bodies which stained with eosin and had a round nucleus. They resembled the cells seen in the angle of the anterior chamber to which reference has been made. Many of the nuclei showed division. The bodies of the nodules were composed of cells, whose cytoplasm was almost filled by a nucleus, and were concentric, elongated ovals, or irregular in shape. They were arranged concentrically in nearly all instances where the nodule was other than small. In all there 
was pigment, sometimes so much as to make the prominence look black. The pigment was in two forms, either intracellular and composed of fine granules, like the pigment in the iris stroma, or forming rounded agglomerations, and amorphous in type (Fig. 4). The nodules were apparently firm in consistence, as it was rare to find one cut by the knife : they were pushed aside and retained their spherical shape. The cells forming the centres of the nodules were also found in large numbers all over the front surface of the iris. Scattered through the iris were other small oval cells with no bodies that one could discern, staining a brighter blue, and forming clusters in the sphincter area in front of the muscle, and in the inner part of the ciliary body. In some sections the ciliary cluster was very noticeable, and the cells could be seen streaming away from it in lines into the iris root, into the back of the ciliary body and forwards to the canal of Schlemm (Figs. 3, 5 and 6). Their arrangement suggested that the primary focus of the trouble might be in the ciliary body, and that one was dealing with a neoplastic condition arising there and secondarily spreading into the iris. This would at least be an understandable explanation.

After much examination I have, however, come to the conclusion that these cells are not small round sarcoma cells, but small lymphocytes, and the case as a whole must therefore be inflammatory in origin, though it becomes much more obscure in interpretation.

Up to the time when one could no longer get the cornea clear by the use of eserine to lower tension, it is certain that no inflammatory changes were present which could be detected by careful slit-lamp examination, yet evidences are brought out by the microscope that slight inflammation had been present. In the root of the iris were to be seen a few plasma-staining cells, and a smaller number whose bodies were full of eosinophile granules. In the part of the iris which was adherent to the cornea the vessels showed hyaline thickening of the coats. The iris contained numerous clump cells, and there were balls of pigment free in the angle of the anterior chamber. Besides these points there were the clusters of cells in the ciliary body, and sphincter area, and lastly the hyaline membrane.

It is a curious point that up to the end I was sure that the only adhesions of the iris to the cornea were on the outer side and above, and both some distance from the pupil, yet the sections show an adhesion of the edge of the pupil above, which is packed with lymphocytes and is covered by an extra thick hyaline coating.

It seems agreed that the formation of a hyaline membrane on the iris is a sequence in cases of chronic glaucoma. In this case, however, it must have been in existence before glaucoma occurred, as it forms part of the prominences which were well marked when 


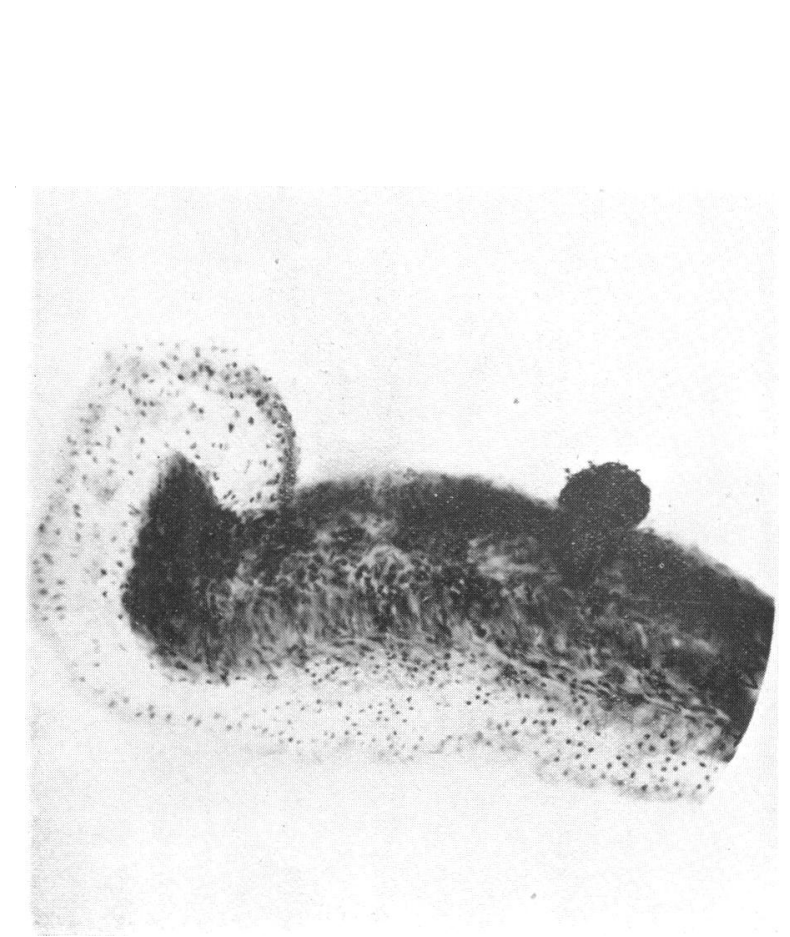

FIG. 1.

Bleached section. A darkly stained nodule. Deeply stained oval nuclei in front of sphincter muscle. Muscle and urea are bent round this layer of cells. Zeiss C.X5.

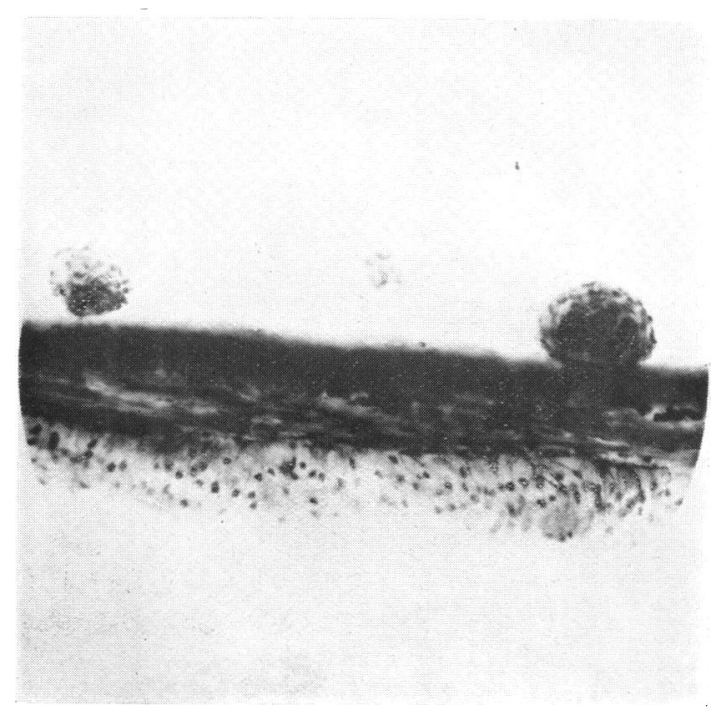

FIG. 2.

Bleached Section. Typical nodules. Zeiss C. $\times 5$. 


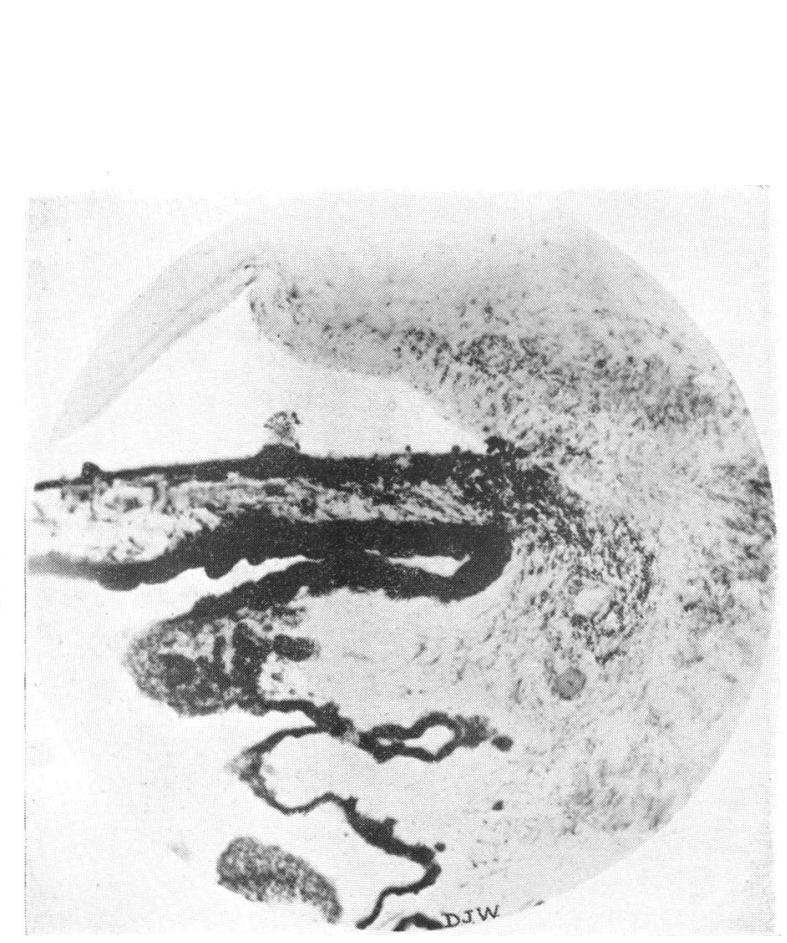

FIG. 3.

Angle of anterior chamber Zeiss A $\times 5$. A large nodule. Balls of pigment in angle. Pigmented stroma cells and small oval cells streaming from pigmented plaque in to ciliary body. Unbleached.

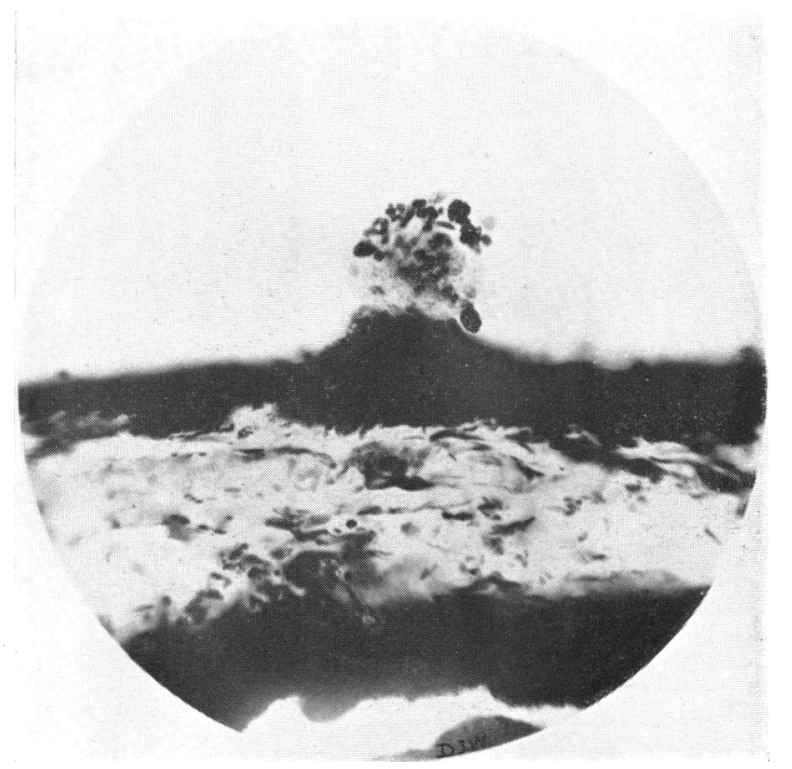

FIG. 4.

Nodule in Fig. 3 Zeiss D.D. $\times 5$. The larger dark spheres are agglomerations of amorphous pigment. Unbleached. 


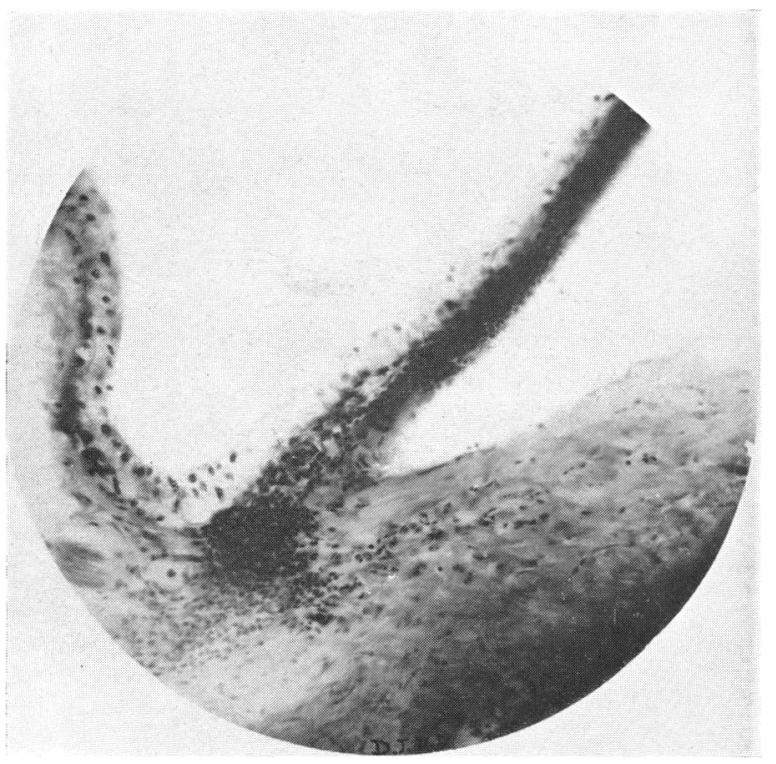

FIG. 5.

Cell cluster in ciliary body. Cells passing in lines into the iris. Zeiss AA $\times 10$. Bleached.

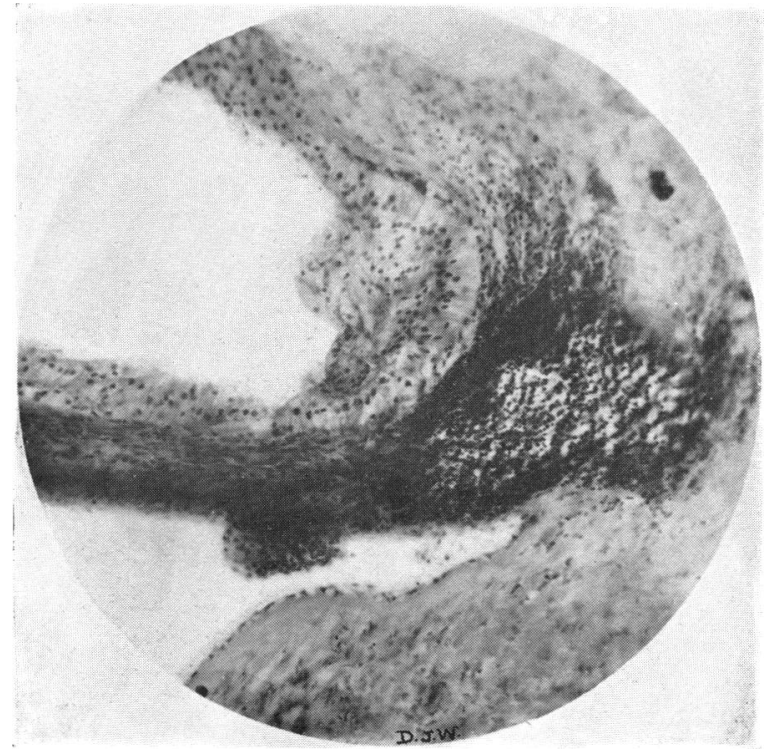

FIG. 6.

Large cell cluster in ciliary body. Cells streaming into posterior part of ciliary body. Endothelium in angle of A.C. Bleached. 


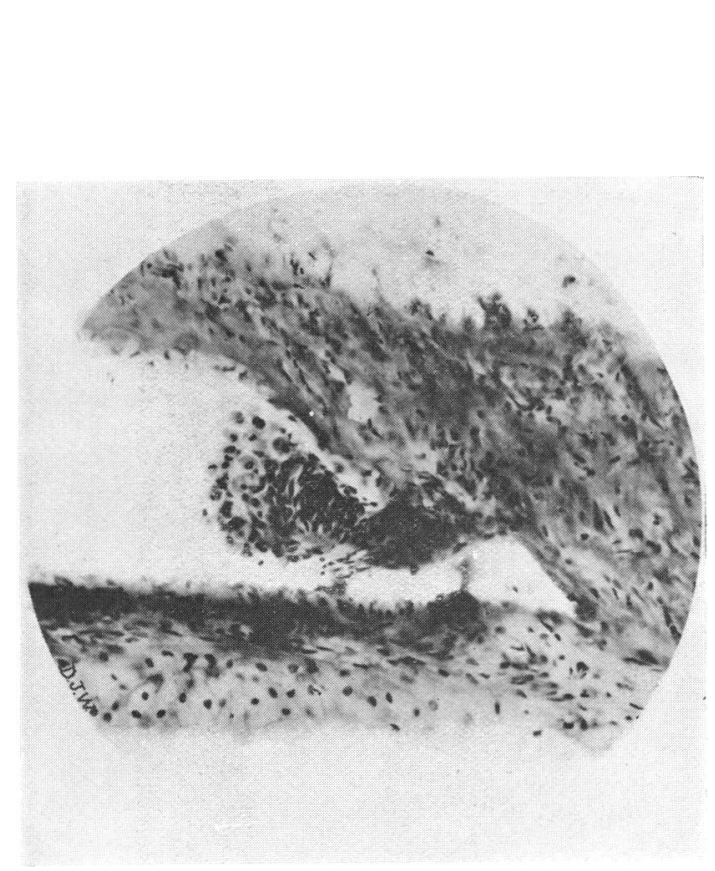

FIG. 7.

Nodule attached to the pectinate ligament and ? to iris. Superficial cells show rounded bodies staining with eosin. Deeper cells show no bodies. Stain well with haemalum.

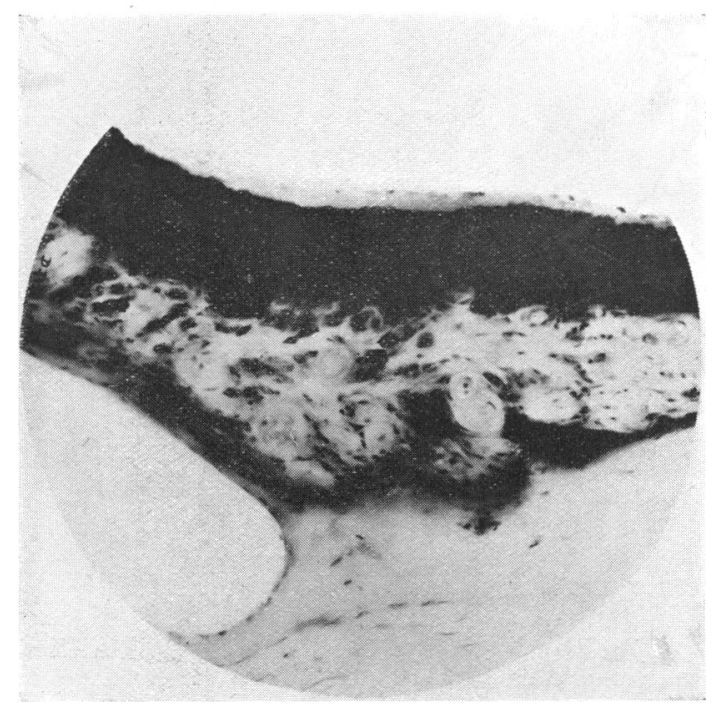

FIG. 8

Section through iris adhesion. Hyaline membrane passing on to cornea, and also on posterior surface of iris. Thickening of walls of blood vessels. 
the case was first seen. The nature of the cells forming the prominences is another problem. Some have bodies which stain readily with eosine and have a small round nucleus. Are these as it would seem endothelial? If so, what are the other cells which show practically no body but which are similar to the large numbers of cells seen on the iris surface and which ought to be endothelial ?

I have sent some of the sections to Mr. Treacher Collins with a request to review the case and add his opinion.

\section{Remarks by E. Treacher Collins}

In complying with Dr. Wood's request, to write my opinion concerning the sections he has been good enough to send me of this very remarkable case, it will, I think, be well to consider separately the several different features which it presents, and then endeavour to ascertain their relation to one another.

Its several different features are :

(1) The presence of a layer of hyaline tissue, like that of Descemet's membrane, on the surface of the iris.

(2) The abnormal deep pigmentation of a sector of the iris.

(3) The rounded nodules on the surface of the iris.

(4) The localized groups of closely packed cells, at the pupillary border of the iris, and at its junction with the ciliary body.

(1) It is now generally agreed that the hyaline layer of Descemet's membrane is the product of the endothelial cells lining it (Roy. Lond. Ophthal. Hosp. Reps., Vol. XIV, p. 305, 1896). In my Erasmus Wilson Lectures at the Royal College of Surgeons (Lancet, February, 1900) I pointed out that pathological formations of such hyaline membranes occurred :.(a) after perforating wounds of the cornea, where the gap left by the retraction of the divided ends of Descemet's membrane becomes bridged across, first by endothelial cells, and later by a new formed hyaline layer; $(b)$ in cases of anterior synechia of the iris, formed either by perforation of the cornea or as the result of a failure in separation of the iris from the cornea in foetal life, where a new formation of a hyaline membrane extends over the surface of the iris from the seat of the adhesion with endothelial cells on its anterior surface; $(c)$ in cases of long standing glaucoma, where the angle of the anterior chamber has been blocked for some time by adhesion of the root of the iris to the back of the cornea, and where a new formed hyaline membrane is found to spread round the angle of the chamber on to the anterior surface of the iris, endothelial cells being present on its anterior surface.

In this article I suggested that the endothelium of the.iris seemed to have acquired the function of forming a hyaline membrane like that of the endothelium of Descemet's membrane. 
A reconsideration of these cases makes me think it more probable that there is, in the first place, an extension of the endothelium of Descemet's membrane on to the surface of the iris along the course of the anterior synechia, and that it is this endothelium which is responsible for the development of the hyaline membrane, and not that on the anterior surface of the iris. Wood's case shows definitely that an extension of endothelium must have preceded the formation of a hyaline membrane, for in it, there is not only a newly-formed hyaline membrane lined by endothelium on the anterior surface of the iris, but. an extension of it round the pupillary border of the posterior surface, where no endothelium normally exists.

At the site of one of the adhesions of the iris to the cornea in Wood's case, which is situated in the position of the small circle of the iris, there is a considerable thickening of hyaline tissue, equal to five or six layers of superimposed Descemet's membrane. Hyaline tissue of this character is very slow in formation, and both this thickened mass, and the new layer on the surface of the iris, must have taken a considerable time to develop. There is no sign of there having been any perforation of the cornea at the seat of the adhesion or of any past inflammation. I think it is most probable that the adhesion is due to a congenital failure in separation of the iris from the cornea. In a case of my own which had a congenital anterior synechia of the iris near the pupillary border, and congenital buphthalmos, there was a thickened hyaline mass, similar to that in Wood's case, and a layer of hyaline tissue on the anterior surface of the iris.

(2) In a case recorded by Dr. A. B. Rees (Amer. Jl. of Ophthal., Vol. VIII, p. 863, 1925) of melanosis of the eye he describes the iris as showing in section, microscopically, a ribbon of pigment anteriorly, so dense and thick that it resembles the pigment on the posterior surface of a normal iris. In Wood's case there is a similar dense pigmentation of the anterior layers of the iris over the melanotic sector. In specimens which I have examined of melanosis of the iris (Trans. Ophthal. Soc. U.K., Vol. XLVI, p. 104) there was also dense pigmentation of its anterior layers, its endothelium and the cells of its anterior limiting layer. Rees also describes how he found in the stroma of the iris in his case, chromatophores which had "acquired so much pigment that they are globular in shape, and show no pigmented processes as seen in typical chromatophores." He compares these globular pigmented cells to "clump cells," and says that all gradations can be seen between them and the normal branching chromatophores. Near the root of the iris in the melanotic area in Wood's case, clump cells can be seen both in the stroma and in its anterior layers, and all gradations can be traced between them and darkly pig- 
mented chromatophores. These gradations in the formation of "clump cells" support Ginsberg's view that they originate in chromatophores, rather than Elschnig's and Lauber's that they are derived from the pigment epithelium. On this matter I was able to quote some very strong evidence in the discussion on Intraocular Melanomata at the Ophthalmological Society in 1926.

There is nothing in the history of Wood's case to show how long the melanotic area had been present, but in view of what is known with regard to similar patches in other cases, I think in all probability it must have dated from birth or early infancy.

(3) Several cases of melanosis of the iris have been recorded in which prominences, of various sizes and shapes, have been seen clinically on the surface of the iris. In Wood's case the prominences are more globular than in those previously described. Histological examination in two of the previously described cases seemed to show that they were mainly composed of endothelial cells, but it is always very difficult to differentiate the cells of the anterior limiting layer of the iris from the endothelium. In Wood's case the round projections seem to be made up partly of endothelial cells and partly of a smaller variety of cells. Some of them are deeply pigmented and others less so. Those situated over the melanotic area-for histologically some are found there though they were not visible clinically-are very deeply pigmented, and in some instances contain clump cells. The nodules in the sections appear frequently as detached circular groups of cells lying superficial to the hyaline layer on the surface of the iris, others, however, have a definite pedicle which passes through the hyaline membrane, so that the cells of which they are composed are continuous with those of the tissue of the iris. As Wood aptly says some of them appear raised up like golf balls on a tee. The detached appearance of some of the nodules is probably due to the narrowness of their pedicle which is not cut across in some of the sections. The situation of these nodules on the anterior surface of the iris suggests that possibly they may be connected with some persistence of the irido-pupillary membrane, beneath which the iris grows in foetal life. Wood notes that he saw clinically some remains of pigment on the lens suggestive of persistent pupillary membrane. If these prominences on the surface of the iris are remnants of the irido-pupillary membrane, then the hyaline membrane which has formed on the surface of the iris has failed to spread over them, though it has formed around them. Possibly its formation may help to account for the unusual globular shape of the excrescences.

(4) Melanosis of the iris has several times been noted as occurring in association with pigmented naevi of the skin of the eyelids or of the face. In such pigmented naevi of the skin there 
are frequently found, besides pigmented cells, unpigmented cells of an embryonic type. It is in such patches of embryonic tissue that malignant growths are liable to originate. In Wood's case there are closely packed groups of unpigmented cells of an embryonic type at the pupillary margin of the iris, where it is adherent to the cornea, and at its junction with the ciliary body. In some of the sections these cells appear to be mostly round in shape, but some spindle-shaped ones are seen to be intermingled with them. In other sections the group of cells at the pupillary margin appears to be mostly composed of spindle-shaped cells. Where round and spindle-shaped cells appear to be mixed together in a section it is always uncertain if the apparent round cells may not really be spindle cells cut transversely. These accumulations of cells in Wood's case do not appear to be inflammatory, and certainly the spindle-shaped cells are not of that nature. Whether or not they are areas commencing to take on malignant activity cannot be certainly determined by histological examination alone.

At the seat of the adhesion of the pupillary margin of the iris to the cornea there seems to be some abnormal distribution of the blood-vessels of the iris, and also, as Wood, remarks a marked sclerosis of some of the vessel walls.

Such being the several different features of interest in this case, in what relation do they stand to one another? A general review of them seems to point to a primary congenital defect in connection with the irido-pupillary membrane; resulting in its imperfect separation from the back of the cornea, so producing the anterior synechiae; and persistence of portions of it forming protrusions on the surface of the iris. There must also have been a formation of a congenital naevus, resulting in the production of the abnormal area of pigmentation and areas of embryonic tissue. The endothelium of Descemet's membrane, due to the anterior synechiae, spread on to the surface of the iris and formed there an abnormal hyaline layer. This hyaline layer extending round the angle of the anterior chamber in a portion of its extent, as well as over the surface of the iris, must have caused some hindrance to the normal channels of exit for fluid from the eye, and so predisposed the eve to glaucoma. It is remarkable that symptoms of glaucoma did not appear earlier than they did. Possibly the areas of embryonic tissue were commencing to grow and manifest malignant tendency, and this may have accounted for the recent onset of glaucomatous symptoms, which led up to the excision of the eye. 